Revista de Psicología Vol. 32 (2), 2014 (ISSN 0254-9247)

\title{
Sentido de pertenencia y compromiso organizacional: predicción del bienestar
}

\author{
Celeste Dávila de León ${ }^{1}$ y Gemma Jiménez García ${ }^{2}$ \\ Universidad Complutense de Madrid
}

Los objetivos del presente trabajo son determinar la validez discriminante del sentido de pertenencia y de los diferentes tipos de compromiso organizacional, y analizar la relación y capacidad predictiva de estos constructos del bienestar subjetivo y psicológico. 354 personas cumplimentaron un cuestionario que permitía medir los constructos descritos. Los resultados muestran que el sentido de pertenencia y los diferentes tipos de compromiso organizacional son constructos diferentes, y que se asocian de forma diferencial con los diversos indicadores del bienestar en función del tipo de puesto de trabajo estudiado. El compromiso afectivo es el predictor más importante para los empleados que desempeńan puestos de base, y el sentido de pertenencia es el único predictor para los puestos de mando. Palabras clave: sentido de pertenencia, identificación organizacional, compromiso organizacional, bienestar.

\section{Sense of belonging and organizational commitment: prediction of well-being}

This following study aimed to determine the discriminant validity of Sense of Belonging and the different types of organizational commitment, and analyse the relationship and the predictive capacity of these constructs for subjective and psychological well-being. 354 people completed surveys measuring the constructs described. The results showed that Sense of Belonging and the different types of organizational commitment are different constructs, and they have different relationships the diverse indicators of well-being according to the type of employment. The affective commitment was the most relevant predictor in entry level employees, and Sense of Belonging was the only predictor in employees with leadership positions.

Keywords: Organizational commitment, sense of belonging, organizational identification, well-being.

1 Doctora en Psicología y profesora en la Universidad Complutense de Madrid. Dirección postal: Dpto. de Psicología Social. F. de CC. Políticas y Sociología, Universidad Complutense de Madrid. Campus de Somosaguas, s/n, 28223, Madrid, España. Contacto: mcdavila@cps.ucm.es.

2 Doctora en Psicología y directora de la Unidad de Psicomotive del Grupo Empresarial GSS. Dirección postal: Guzmán el Bueno, 133. Ed. Germania, Planta 6a, 28003, Madrid, España. Contacto: gjimenez@grupogss.com. 
Tradicionalmente el análisis de la vinculación psicológica existente entre la organización y los miembros se ha centrado fundamentalmente en el estudio del compromiso organizacional. Actualmente, la mayoría de los investigadores creen que el compromiso organizacional es una actitud multidimensional (Meyer, Allen \& Topolnysky, 1998). En los últimos años, el modelo de Meyer y Allen (1991) ha dominado la investigación en torno a este concepto. Estos autores distinguen entre compromiso afectivo (CA, recoge el deseo de seguir perteneciendo a la organización, alude a la vinculación afectiva con la organización), compromiso normativo $(\mathrm{CN}$, referido al sentido de obligación hacia la organización) y compromiso de continuación (CC, continúan en la organización porque necesitan hacerlo). En algunas investigaciones el último tipo de compromiso tiende a estudiarse diferenciando dos facetas, por un lado, la necesidad de continuar en base a los costes asociados a dejar la organización (CCS, alto sacrificio) y, por otro lado, la necesidad de continuar debido a la falta de alternativas de empleo disponibles (CCF) (Panaccio \& Vandenberghe, 2009; Stinglhamber, Bentein \& Vandenberghe, 2002, por ejemplo).

Otros constructos también pueden permitir explicar la vinculación psicológica de los empleados con la organización, como es el caso del sentido de pertenencia. Maslow (1954) describió la pertenencia como una necesidad básica humana. Anant (1966) define la pertenencia como el sentido de implicación personal en un sistema social, de tal forma que la persona sienta que es una parte indispensable e integral de ese sistema. En base a esta definición, Hagerty, Lynch-Sauer, Patusky, Bouwsema y Collier (1992) identifican como elementos esenciales para desarrollar un sentido de pertenencia la experiencia de sentirse valorado, necesitado y aceptado por otras personas, grupos o ambientes; y la percepción por parte de la persona de que sus características son similares o complementan a las de las personas que pertenecen al sistema, es decir, 
la experiencia de ajustarse o de ser congruente con otras personas, grupos o ambientes a través de características compartidas o complementarias. Cameron (2004) considera al sentido de pertenencia, o lo que él denomina "vínculos interpersonales", como una faceta más de la identidad social y, en relación al ámbito organizacional, como una faceta de la identificación organizacional (Harris \& Cameron, 2005).

Aunque el análisis de las diferencias y similitudes entre el concepto de compromiso organizacional e identificación organizacional no es nuevo (Ashforth \& Mael, 1989; Gautam, Van Dick \& Wagner, 2004; Lisbona, Morales \& Palací, 2006; Riketta, 2005; Van Knippenberg \& Sleebos, 2006), el estudio concreto de la comparación entre compromiso organizacional y sentido de pertenencia sí lo es. Hasta el momento, la mayor parte de la investigación sobre sentido de pertenencia se ha centrado en estudiantes con relación a sus centros educativos o algún elemento de los mismos como por ejemplo, la clase o el grupo de pares, prestando atención a sus implicaciones de cara a la permanencia de los estudiantes en los centros y a aspectos como su motivación, rendimiento académico y manifestación de problemas de conducta (Johnson et al., 2007, por ejemplo). Pero el estudio del sentido de pertenencia en el ámbito laboral resulta algo bastante novedoso. El único estudio que hasta ahora se ha desarrollado es el de Dávila y Jiménez (2012). Dicho estudio apoya la validez discriminante del sentido de pertenencia $y$, concretamente, del compromiso afectivo, encontrando diferentes relaciones de ambos constructos con diversos tipos de apoyo percibido, el ajuste de valores entre el empleado, el supervisor y la organización, el comportamiento de ciudadanía organizacional y la intención de continuar en el futuro en la organización. En términos generales, se halla que el concepto de compromiso organizacional, al menos el afectivo, parece ser más útil en la predicción de comportamientos que pueden ser deseables para la organización. Sin embargo, dada la escasez de estudios que planteen objetivos similares, resulta bastante arriesgado rechazar en base a tan escasa evidencia la potencial relevancia del sentido de pertenencia en el estudio del comportamiento organizacional. 
Con el objetivo de seguir profundizando en las potenciales diferencias y similitudes entre el concepto de sentido de pertenencia y compromiso organizacional, se plantea la siguiente investigación, la cual trata de aportar nueva evidencia empírica sobre la validez discriminante de ambos constructos, en este caso abordando el estudio de otros tipos de compromiso organizacional, $\mathrm{y}$, al mismo tiempo, trata de analizar la capacidad predictiva de cada uno de estos conceptos de otras variables, comportamientos o estados que pueden ser deseables tanto para los empleados como para la organización. En este sentido, y siguiendo lo que en su momento apuntó Hagerty, Wiliams, Conye y Early (1996) sobre que el sentido de pertenencia es un elemento importante para la salud mental y el bienestar social, que se asocia al funcionamiento psicológico y social, se presenta el análisis del bienestar de los empleados.

El bienestar ha sido definido como "una experiencia y funcionamiento psicológico óptimo" (Ryan \& Deci, 2001, p.142), y pueden identificarse dos orientaciones en su estudio: el bienestar subjetivo y el bienestar psicológico. Diener, Suh, Lucas y Smith (1999, p.277) definen el bienestar subjetivo como una "amplia categoría de fenómenos que incluyen: las respuestas emocionales, la satisfacción en diferentes dominios o áreas y un juicio global de satisfacción vital". De esta manera, se ha considerado un constructo multidimensional, que incluye componentes cognitivos, como la satisfacción vital o la satisfacción laboral, y componentes emocionales, como el afecto positivo y el afecto negativo.

Otra tradición de estudio más reciente en torno al bienestar se ha centrado en el bienestar psicológico, que incluye componentes cognitivos, motivaciones y conductuales, y alude en mayor medida a aspectos como el crecimiento personal y el desarrollo del potencial humano (Culberton, Fullagar \& Mills, 2010). Esta otra tradición se refiere a los principales indicadores del funcionamiento positivo (Díaz et al., 2006).

El estudio del bienestar subjetivo y del bienestar psicológico supone dos concepciones del bienestar teóricamente relacionadas pero empíricamente diferenciadas, ya que se basan en diferentes indicadores de medida (Blanco \& Díaz, 2005). El bienestar psicológico puede 
ser entendido como un estilo de vida que permite obtener bienestar subjetivo, que podría considerarse como un estado mental positivo. Pero mientras que el bienestar psicológico podría ser una condición suficiente aunque no necesaria para experimentar bienestar subjetivo, el bienestar subjetivo no sería una condición ni suficiente ni necesaria para experimentar bienestar psicológico (Meyer \& Maltin, 2010).

La salud y la experiencia de bienestar en el lugar de trabajo ha recibido bastante atención por parte de la investigación organizacional desde diferentes perspectivas. Se ha encontrado que el bienestar puede potencialmente afectar a los trabajadores y a las organizaciones de diversas formas. Por ejemplo, cuando los trabajadores experimentan pobre salud y bienestar en el trabajo pueden ser menos productivos, disminuir de forma consistente sus contribuciones generales a la organización, tomar decisiones de menor calidad, y ser más propensos al absentismo. Sumado a ello, a pesar de que las personas dedican solo cerca de un tercio de las horas del día al trabajo, no necesariamente dejan el trabajo cuando abandonan la oficina o el centro. El trabajo y el resto de las áreas vitales se encuentran interrelacionadas, por lo que el bienestar experimentado en el trabajo no solo tiene implicaciones en ése área de nuestras vidas, sino que también puede influir en cómo uno se siente en el resto de sus áreas vitales (Danna \& Griffin, 1999). En los últimos años, ha habido un creciente interés en adoptar una aproximación positiva en el análisis de las organizaciones y de la conducta organizacional, más que intentar predecir consecuencias negativas para los individuos o las organizaciones (abandono, burnout, absentismo, etc.) los investigadores han empezado a examinar formas en las que se pueden obtener consecuencias positivas y a identificar qué factores dan lugar a ellas (Culbertson et al., 2010). El bienestar debe ser visto como algo más que la ausencia de enfermedad, y resulta indispensable incluir en los estudios índices de funcionamiento óptimo o crecimiento personal, por ejemplo, junto con los índices relativos al estrés psicológico o a la enfermedad física (Meyer \& Maltin, 2010).

La investigación previa ha mostrado que el CA se relaciona con el bienestar de los empleados (Meyer \& Maltin, 2010; Meyer, Stanley, 
Hercovitch \& Topolnytsky, 2002; Panaccio \& Vandenberghe, 2008, por ejemplo), concretamente de forma positiva con el bienestar físico y la salud (Bridger, Kilminster \& Slaven, 2007; Siu, 2002), la salud mental (Grawitch, Trares \& Kohler, 2007), la satisfacción laboral (Cooper-Hakim \& Viswesvaran, 2005; Lisbona et al., 2006), la satisfacción vital (Zickar, Gibby \& Jenny, 2004; Lu, Siu, Spector \& Shi, 2009) y el afecto positivo (Thorensen, Kaplan, Barsky, Warren \& De Chermont, 2003), y de forma negativa con el afecto negativo (Thoresen et al., 2003) y con aspectos como la depresión, el cansancio emocional o el estrés, por ejemplo (Epitropaki \& Martin, 2005; Lambert, Hogan \& Griffin, 2008).

Con relación al $\mathrm{CN}$, los resultados son escasos y no terminan de ser concluyentes. Mientras que algunos estudios muestran que no mantiene una relación significativa con el bienestar (Addae \& Wang, 2006; Somers, 2009), otros, como es el caso de Cooper-Hakim y Viswesvaran (2005), de Lisbona y colaboradores (2006), y de Tan y Akhtar (1998), hallan una asociación positiva con la satisfacción laboral en el primer y segundo caso, y una relación también positiva con el cansancio emocional en el tercer caso. También son relativamente escasos los estudios que se centran en el CC, algunos de ellos muestran relaciones negativas con la satisfacción vital (Zickar et al, 2004) y con la satisfacción laboral (Meyer et al, 2002), y en general con el bienestar (Panaccio \& Vandenberghe, 2008), otros estudios hallan relaciones positivas con índices de tensión laboral, ansiedad y cansancio emocional, por ejemplo (Addae \& Wang, 2006; Bakker, Demerouti, de Boer \& Schaufeli, 2003; Irving \& Coleman, 2003).

En resumen, no se encuentra evidencia que sugiera que el CA tiene implicaciones negativas de cara al bienestar, sino que al contrario, se sugiere que un fuerte compromiso organizacional afectivo podría tener beneficios positivos para la salud. Aunque los estudios sobre el resto de componentes del compromiso organizacional son menos comunes, en ellos el CC parece estar positivamente relacionado con el estrés o la tensión, y en el caso del CN las relaciones existentes parecen ser muy débiles (Meyer \& Maltin, 2010). Hasta la fecha, la mayor parte 
de la investigación se ha centrado en el estudio de la relación con el bienestar subjetivo, pero Meyer y Maltin (2010) sugieren que también el compromiso afectivo podría asociarse positivamente al bienestar psicológico. En este sentido, los estudios que muestran la existencia de una relación entre CA y engagement (Hallberg \& Schaufeli, 2006), por ejemplo, irían en esa línea. Se trata de un constructo considerado como una consecuencia del bienestar psicológico y en algunos casos también se le ha considerado un indicador del mismo (Meyer \& Maltin, 2010), aunque otros autores abogan por considerarlo un constructo teóricamente diferente al bienestar psicológico (Culberton et al., 2010).

Con relación al sentido de pertenencia, una asunción fundamental de la Teoría de la Identidad Social (Tajfel \& Turner, 1979) describe que la identificación con los grupos sirve para mantener un autoconcepto positivo y, de esta forma, se asociaría al bienestar. Pero hay pocos estudios que hayan analizado el impacto de la identificación organizacional sobre el bienestar. Por ejemplo, Van Dick y Wagner (2002) hallaron una relación negativa entre identificación organizacional y dolencias de salud física. Schaubroeck y Jones (2000) mostraron que la identificación organizacional podría funcionar como un amortiguador contra los estresores organizacionales. Brotheridge y Lee (2003) encontraron una relación negativa con la disonancia emocional y con la experiencia de burnout, y Wegge, van Dick, Fisher, Wecking y Moltzen (2006) encontraron que la identificación organizacional era un predictor independiente de la satisfacción laboral y del bienestar. En conclusión, la evidencia disponible muestra la existencia de una influencia positiva de la identificación organizacional sobre el bienestar. A pesar de estos datos, hasta el momento ninguna investigación ha tratado de analizar la relación concreta entre sentido de pertenencia y bienestar en el ámbito laboral. Las únicas investigaciones analizan tal asociación en otros ámbitos. Por ejemplo, Young, Russell y Powers (2004) relacionaron el sentido de pertenencia al vecindario con variables como la salud física y mental, y el estrés en mujeres mayores. Ron (2004) analizó la relación entre sentido de pertenencia y síntomas de depresión y ansiedad en personas indigentes. Mock, Fraser, Knutson y Prier (2010) encontraron 
que el sentido de pertenencia mediaba parcialmente la asociación entre la práctica de actividades físicas de tiempo libre y la salud mental y física en adultos con artritis reumatoide. McCallum y McLaren (2011) hallaron que el sentido de pertenencia a la comunidad general contribuyó significativamente a la predicción de la depresión en personas homosexuales. Finalmente, Kitchen, Williams y Chowhan (2012) encontraron una asociación consistente y significativa entre sentido de pertenencia a la comunidad y salud controlando el estatus geográfico y socioeconómico. En general, los resultados hallados parecen mostrar la existencia de una relación entre sentido de pertenencia y bienestar.

En base a lo descrito hasta el momento se plantea analizar la capacidad predictiva del compromiso organizacional y del sentido de pertenencia de indicadores tanto del bienestar subjetivo (satisfacción vital, afecto positivo, afecto negativo y satisfacción laboral) como del bienestar psicológico. Con relación al bienestar psicológico se plantea analizar el engagement. Aunque no existe mucho consenso respecto a cuál es su significado y cuál es la mejor forma de evaluarlo, ha sido definido como un estado mental positivo, de realización en el trabajo, que se caracteriza por altos niveles de energía y resiliencia mental en el desarrollo del mismo, y por el deseo de esforzarse incluso ante la presencia de dificultades; alta implicación laboral y sentimientos de significación, entusiasmo, inspiración, orgullo y reto por el trabajo; y una total concentración en el desarrollo del trabajo (el tiempo pasa rápidamente y resulta difícil desconectar del mismo) (Salanova \& Schaufeli, 2009). Sumado a ello, partiendo de la idea de que en ocasiones ciertos factores contextuales pueden incidir en las relaciones establecidas entre variables (Cambré, Kippers, van Veldhoven \& de Witte, 2012; Kavanagh \& Halpern, 1977, por ejemplo) se optó por llevar a cabo dichos análisis en dos grupos diferentes de empleados: empleados que desempeñaban puestos considerados de mando (supervisores, coordinadores, etc.) y trabajadores que desempeñaban puestos considerados de base. 


\section{Método}

\section{Participantes}

Los participantes en el estudio fueron un total de 354 trabajadores españoles pertenecientes al Grupo GSS, que es una empresa especializada en servicios de contact center. El 15\% de los empleados de dicha empresa participaron en el estudio y todos ellos formaban parte del Departamento de Operaciones. El $80.5 \%$ eran mujeres y el $18.9 \%$ varones. La media de edad era 36.54 años $(D T=9.10)$. El $9.3 \%$ tenía estudios primarios, el $56.8 \%$ secundarios y, finalmente, el $33.6 \%$ universitarios. Prácticamente la mitad trabajaba a tiempo completo (51\%).

El $18.9 \%$ de estos trabajadores desempeñaban puestos considerados de mando o coordinación, eran responsables directos o superiores inmediatos de los agentes teleoperadores (puestos considerados de base), y el $81.1 \%$ desempeńaban puestos de agente teleoperador. A continuación se describe el perfil de cada uno de estos grupos:

En el grupo de agentes, el $80.8 \%$ eran mujeres y el 18.5\% varones, y la media de edad era 36.46 años $(D T=9.57)$. Con relación al nivel de estudios, el $11.1 \%$ tenía estudios primarios, el 56.8\% secundarios, y el $31.7 \%$ universitarios. El 58.9\% trabajaba a tiempo parcial.

En el grupo de mandos, el $79.1 \%$ eran mujeres y el $20.9 \%$ varones. La media de edad era 36.91 años $(D T=6.73)$. El $1.5 \%$ tenía estudios primarios, el $56.7 \%$ secundarios y el $41.8 \%$ universitarios. La mayoría trabajaba a tiempo completo $(94 \%)$.

\section{Instrumentos}

Los participantes en el estudio cumplimentaron un cuestionario donde se recogían las siguientes medidas:

Compromiso organizacional. Para evaluar cada una de las dimensiones de este constructo se utilizó una adaptación al español de la versión de Bentein, Vandenberg y Vandenberghe y Stinglhamber (2005) de la escala de Meyer y Allen (1991). Para llevar a cabo dicha adaptación se tradujo la escala original al español, y una persona bilingüe en inglés 
y español evaluó la equivalencia del contenido de cada ítem. En esa adaptación se realizaron algunos cambios para ajustar los ítems a las expresiones habituales en español. Este instrumento incluye un total de 18 ítems (cada subescala incluye 6 ítems, con relación al CC, 3 ítems permiten evaluar la faceta de alto sacrificio y 3 la de falta de alternativas) con un formato de respuesta tipo Likert que va de 1 (completamente en desacuerdo) a 7 (completamente de acuerdo). La consistencia interna obtenida para cada subescala fue 0.92 (CA), 0.87 (CN), 0.69 (CCS), 0.69 (CCF).

Sentido de pertenencia. En este caso se utilizó una adaptación al español y al ámbito organizacional de siete de los 18 items que componen la escala desarrollada por Hagerty y Patusky (1995) (Dávila \& Jiménez, 2012) para evaluar el estado psicológico del sentido de pertenencia. Dicha escala tiene un formato de respuesta que va de 1 (totalmente en desacuerdo) a 7 (totalmente de acuerdo). Las altas puntuaciones en este constructo se interpretan como un bajo sentido de pertenencia. El índice de consistencia interna obtenido fue 0.88 .

Bienestar. Se midieron 4 dimensiones del bienestar subjetivo y un indicador del bienestar psicológico.

$\mathrm{El}$ afecto positivo y negativo era evaluado usando una adaptación al español de la escala PANAS de Watson, Clark y Tellegen (1988). Para dicha adaptación se siguió el procedimiento descrito previamente. La escala original consta de 20 ítems que aluden a sentimientos o emociones que se pueden experimentar hacia el trabajo, 10 de esos ítems aluden a emociones positivas y 10 a emociones negativas, pero se decidió eliminar uno de los ítems de la subescala de afecto positivo, ya que tenía un elevado peso factorial muy similar en ambas subescalas. El formato de respuesta es una escala de 7 puntos que va de 1 (muy ligeramente o no del todo) a 7 (extremadamente). El índice de consistencia interna para la subescala de afecto positivo fue de 0.86 y para la subescala de afecto negativo de 0.85 .

La satisfacción vital se evaluó utilizando la adaptación realizada por Atienza, Pons, Balaguer y Garcia-Merita (2000) de la escala de Diener, Emmons, Larsen y Griffin (1985). Dicha escala está compuesta 
por 5 ítems y el formato de respuesta utilizado es una escala tipo Likert de 7 puntos, donde 1 es igual a "absolutamente en desacuerdo" y 7 a "absolutamente de acuerdo". Estudios previos han constatado una adecuada fiabilidad y validez en población española (Díaz \& SánchezLópez, 2002, por ejemplo). El índice de consistencia interna obtenido con esta escala fue de 0.84 .

La satisfacción laboral general era evaluada utilizando una adaptación al español de la escala de Warr, Cook y Wall, (1979), que ha sido utilizada en otros trabajos posteriores (Mullarkey, Jackson, Wall, Wilson \& Grey-Taylor, 1997, por ejemplo). Para dicha adaptación se siguió el mismo procedimiento descrito previamente. La escala está compuesta por 4 ítems con un formato de respuesta tipo Likert de 7 puntos que va de 1 (totalmente en desacuerdo) a 7 (totalmente de acuerdo). El índice de consistencia interna obtenido fue de 0.81 .

El engagement era evaluado con la versión reducida de la escala UWES desarrollada y adaptada al español por Schaufeli, Bakker y Salanova (2006). La escala está compuesta por 9 ítems con un formato de respuesta tipo Likert de 7 puntos, que va de 1 (nunca) a 7 (Siempre). El índice de consistencia interna obtenido para la versión reducida completa fue de 0.90 .

\section{Procedimiento}

Mediante la visita concertada de los investigadores a los diferentes servicios que componen las plataformas telefónicas del Grupo GSS, se aplicó el cuestionario a diferentes grupos no superiores a 15 empleados, formados al azar a lo largo de la jornada laboral y utilizando un tipo de muestreo no probabilístico. La aplicación de los cuestionarios se llevó a cabo en salas de formación habilitadas para este fin. La participación de los empleados en la investigación era totalmente voluntaria y se les informaba que sus datos serían tratados con total confidencialidad, respetando el anonimato de los participantes. 


\section{Resultados}

Para el logro del objetivo propuesto se llevaron a cabo análisis factoriales confirmatorios, análisis de correlaciones, ANOVA de un factor y análisis de regresión múltiple utilizando los paquetes de análisis estadístico IBM SPSS Statistics 19 e IBM SPSS Amos 19.

Con el propósito de analizar la validez discriminante del sentido de pertenencia y de los diferentes tipos de compromiso organizacional se contrastaron tres modelos de medida en cada caso, que variaban en función del número de factores y la correlación o independencia de los mismos. En todos los casos, para el desarrollo de los análisis factoriales confirmatorios se utilizó el método de estimación de máxima verosimilitud. Los índices de bondad de ajuste relativos a cada uno de los modelos se encuentran recogidos en la Tabla 1. Como puede comprobarse, los dos únicos modelos que prácticamente en todos los casos se ajustan de forma aceptable a los datos son el modelo de dos factores ortogonales y el modelo de dos factores relacionados. Con relación al CA, el modelo con los dos factores relacionados presenta unos índices de bondad de ajuste ligeramente mejores y el test de diferencias de $c^{2}$ muestra que tales diferencias son significativas $\left(\Delta c_{(1)}^{2}=20.21\right.$, $p<.01)$. En el caso del $\mathrm{CN}$, también se encuentra una ligera mejoría en el modelo con los dos factores relacionados y el test de diferencias de $c^{2}$ muestra que tales diferencias son significativas $\left(\Delta c^{2}{ }_{(1)}=6.74, p<.05\right)$. Para el CC, las diferencias son mínimas entre el modelo de dos factores independientes y el modelo de dos factores relacionados para la subescala de sacrificio, y el test de diferencias de $c^{2}$ muestra que tales diferencias no son significativas $\left(\Delta c^{2}{ }_{(1)}=.08, p<.05\right)$. Por último, para la subescala de falta de alternativas el modelo de dos factores relacionados presenta un mejor ajuste y el test de diferencias de $c^{2}$ muestra que tales diferencias son significativas $\left(\Delta c_{(1)}^{2}=33.77, p<.01\right)$. En función de estos resultados podría concluirse que la escala de sentido de pertenencia y las escalas de CA, CN y CC (sacrificio y falta de alternativas) evalúan constructos diferentes, aunque estos están relacionados en todos los casos con la excepción del CC basado en el sacrificio, donde el modelo 
más aceptable por ser al más parsimonioso sería el de los dos factores no relacionados.

\section{Tabla 1}

Indices de bondad de ajuste de los diferentes modelos factoriales analizados Modelos $\quad \chi^{2}$ Df $p \quad \chi^{2} / \mathrm{df}$ CFI GFI RMSEA IFI TLI C. Afectivo

\begin{tabular}{llllllllll}
\hline Un único factor & 650.95 & 65 & .00 & 10.01 & .75 & .69 & .16 & .75 & .70
\end{tabular}

Dos factores ortogonales $\begin{array}{lllllllll}205.06 & 65 & .00 & 3.15 & .94 & .91 & .07 & .94 & .92\end{array}$

Dos factores oblicuos $\begin{array}{lllllllll}184.85 & 64 & .00 & 2.88 & .94 & .92 & .07 & .94 & .93\end{array}$

C. Normativo

\begin{tabular}{llllllllll}
\hline Un único factor & 699.84 & 65 & .00 & 10.76 & .59 & .68 & .16 & .59 & .50
\end{tabular}

Dos factores ortogonales $\begin{array}{lllllllll}200.12 & 65 & .00 & 3.07 & .91 & .91 & .07 & .91 & .89\end{array}$

Dos factores oblicuos $\begin{array}{lllllllll}193.38 & 64 & .00 & 3.02 & .91 & .92 & .07 & .91 & .89\end{array}$ C. Continuidad (sacrificio)

\begin{tabular}{llllllllll}
\hline Un único factor & 300.81 & 35 & .00 & 8.59 & .66 & .84 & .14 & .67 & .57
\end{tabular}

Dos factores ortogonales $\begin{array}{lllllllll}107.14 & 35 & .00 & 3.06 & .91 & .94 & .07 & .91 & .88\end{array}$

Dos factores oblicuos $\begin{array}{lllllllll}107.06 & 34 & .00 & 3.14 & .90 & .94 & .07 & .91 & .88\end{array}$

C. Continuidad (falta alternativas)

\begin{tabular}{llllllllll}
\hline Un único factor & 228.18 & 35 & .00 & 6.51 & .76 & .87 & .12 & .76 & .69
\end{tabular}

Dos factores ortogonales $\begin{array}{lllllllll}120.97 & 35 & .00 & 3.45 & .89 & .93 & .08 & .89 & .86\end{array}$

Dos factores oblicuos

$\begin{array}{lllllllll}87.20 & 34 & .00 & 2.56 & .93 & .95 & .06 & .93 & .91\end{array}$


En cuanto a la relación de sentido de pertenencia y los diferentes tipos de compromiso con otras variables, en la Tabla 2 se presentan las correlaciones, y las medias y desviaciones típicas de todas las variables analizadas tanto para el grupo de mandos como para el grupo de agentes. $\mathrm{Al}$ analizar las posibles diferencias en cada una de las variables analizadas entre los dos grupos de empleados a través de ANOVA de un factor se encontró que las diferencias eran significativas en los siguientes casos: $\mathrm{CA}\left(F_{(1,353)}=14.40 ; p<0.01\right), \mathrm{CN}\left(F_{(1,353)}=4.25\right.$; $p<0.05)$, CC de falta de alternativas $\left(F_{(1,353)}=11.89 ; p<0.01\right)$, afecto positivo $\left(F_{(1,353)}=5.87 ; p<0.05\right)$, satisfacción laboral $\left(F_{(1,353)}=13.09\right.$; $p<0.01)$, y engagement $\left(F_{(1,353)}=12.47 ; p<0.01\right)$. En todos los casos excepto en el CC las medias eran superiores para el grupo de mandos.

Los resultados obtenidos para el grupo de agentes (Tabla 2, parte superior) mostraron que existía una correlación significativa entre sentido de pertenencia y dos tipos de compromiso: CA $(r=-.22, p<.01)$ y CC (falta de alternativas) $(r=.22, p<.01)$. Respecto a las variables de bienestar, el sentido de pertenencia se relacionaba de forma significativa con todas ellas: afecto positivo $(r=-.28, \mathrm{p}<.01)$, afecto negativo $(r=.25, p<.01)$, satisfacción vital $(r=-.16, p<.01)$, satisfacción laboral $(r=-.29, p<.01)$ y engagement $(r=-.24, p<.01)$. El CA mostraba una relación significativa también con todas esas variables: afecto positivo $(r=.52 p<.01)$, afecto negativo $(r=-.22, p<.01)$, satisfacción vital $(r=.25, p<.01)$, satisfacción laboral $(r=.71, p<.01)$ y engagement $(r=.70, p<.01)$. Estas variables también se encontraban significativamente relacionadas con el CN (afecto positivo $(r=.46, p<.01$ ), afecto negativo $(r=-.12, p<.05)$, satisfacción vital $(r=.17, p<.01)$, satisfacción laboral $(r=.53, p<.01)$ y engagement $(r=.51, p<.0)$ y con CC relativo a la falta de alternativas (afecto positivo $(r=-.22, p<.01)$, afecto negativo $(r=.25, p<.01)$, satisfacción vital $(r=-.20, p<.01)$, satisfacción laboral $(r=-.23, p<.01)$ y engagement $(r=-.20, p<.0)$. En cambio el CC relativo al sacrificio solo mostró una relación significativa con afecto positivo $(r=.26, p<.01)$, satisfacción laboral $(r=.41$, $p<.01)$ y engagement $(r=.33, p<.0)$. 


\section{Tabla 2}

Medias, desviaciones tipicas y correlaciones para sentido de pertenencia, compromiso organizacional y las variables de bienestar

\begin{tabular}{|c|c|c|c|c|c|c|c|c|c|c|}
\hline \multicolumn{11}{|l|}{ AGENTES } \\
\hline Variables & 1 & 2 & 3 & 4 & 5 & 6 & 7 & 8 & 9 & 10 \\
\hline $\begin{array}{l}\text { 1. Sentido de } \\
\text { pertenencia }\end{array}$ & ---- & & & & & & & & & \\
\hline 2. $\mathrm{CA}$ & $-.222^{* *}$ & ---- & & & & & & & & \\
\hline 3. $\mathrm{CN}$ & -.092 & $.713^{* *}$ & ---- & & & & & & & \\
\hline 4. CCF & $.227^{* *}$ & $-.174^{* *}$ & -.045 & ---- & & & & & & \\
\hline 5. CCS & -.065 & $.546^{* *}$ & $.633^{* *}$ & $.155^{* *}$ & ---- & & & & & \\
\hline 6. Afecto positivo. & $-.283^{* *}$ & $.521^{* *}$ & $.466^{* *}$ & $-.220^{* *}$ & $.269^{* *}$ & ---- & & & & \\
\hline 7. Afecto negativo. & $.258^{* *}$ & $-.225^{* *}$ & $-.126^{*}$ & $.256^{* *}$ & -.066 & -.011 & ---- & & & \\
\hline 8. Satisfacción V. & $-.160^{* *}$ & $.253^{* *}$ & $.172^{* *}$ & $-.205^{* *}$ & .035 & $.268^{* *}$ & $-.295^{* *}$ & --- & & \\
\hline 9. Satisfacción L. & $-.297^{* *}$ & $.719^{* *}$ & $.533^{* *}$ & $-.235^{* *}$ & $.415^{* *}$ & $.559^{* *}$ & $-.385^{* *}$ & $.269^{* *}$ & ---- & \\
\hline 10. Engagement & $-.245^{* *}$ & $.701^{* *}$ & $.514^{* *}$ & $-.202^{* *}$ & $.332^{* *}$ & $.683^{* *}$ & $-.235^{* *}$ & $.273^{* *}$ & $.796^{* *}$ & --- \\
\hline$M$ & 2.16 & 3.40 & 2.92 & 3.46 & 3.64 & 4.69 & 2.66 & 4.51 & 3.95 & 4.34 \\
\hline$\underline{D T}$ & 1.04 & 1.55 & 1.37 & 1.52 & 1.46 & 1.04 & 1.01 & 1.30 & 1.43 & 1.20 \\
\hline \multicolumn{11}{|l|}{ MANDOS } \\
\hline Variables & 1 & 2 & 3 & 4 & 5 & 6 & 7 & 8 & 9 & 10 \\
\hline $\begin{array}{l}\text { 1. Sentido de } \\
\text { pertenencia }\end{array}$ & ---- & & & & & & & & & \\
\hline 2. CA & -.141 & ---- & & & & & & & & \\
\hline 3. $\mathrm{CN}$ & $-.313^{*}$ & $.505^{* *}$ & --- & & & & & & & \\
\hline 4. $\mathrm{CCF}$ & $.565^{* *}$ & -.156 & -.211 & ---- & & & & & & \\
\hline 5. CCS & .151 & $.261^{*}$ & $.245^{*}$ & .178 & --- & & & & & \\
\hline 6. Afecto positivo. & $-.480^{* *}$ & $.288^{*}$ & $.348^{* *}$ & $-.334^{* *}$ & .195 & --- & & & & \\
\hline 7. Afecto negativo. & $.450^{* *}$ & .022 & .043 & $.424^{* *}$ & .135 & -.072 & --- & & & \\
\hline 8. Satisfacción V. & $-.306^{*}$ & .044 & .147 & $-.312^{*}$ & .124 & $.459^{* *}$ & $-.242^{*}$ & ---- & & \\
\hline 9. Satisfacción L. & $-.363^{* *}$ & .160 & $.269^{*}$ & $-.303^{*}$ & .042 & $.459^{* *}$ & $-.290^{*}$ & $.393^{* *}$ & --- & \\
\hline 10. Engagement & $-.449^{* *}$ & $.367^{* *}$ & $.321^{* *}$ & $-.353^{* *}$ & .196 & $.793^{* *}$ & -.057 & $.527^{* *}$ & $.637^{* *}$ & --- \\
\hline$M$ & 1.96 & 4.16 & 3.30 & 2.76 & 3.68 & 5.02 & 2.60 & 4.63 & 4.62 & 4.90 \\
\hline$D T$ & .89 & 1.11 & 1.32 & 1.38 & 1.30 & .89 & .96 & 1.12 & 1.02 & .98 \\
\hline
\end{tabular}

Nota: ${ }^{*} p<.05 ;{ }^{* *} p<.01$. 
Los resultados obtenidos para el grupo de mandos (Tabla 2, parte inferior) mostraron que existía una correlación significativa entre sentido de pertenencia y dos tipos de compromiso: $\mathrm{CN}(r=-.31, p<.05)$ y CC (falta de alternativas) $(r=.56, p<.01)$. Respecto a las variables de bienestar, el sentido de pertenencia se relacionaba de forma significativa con todas ellas: afecto positivo $(r=-.48, p<.01)$, afecto negativo $(r=.45$, $p<.01)$, satisfacción vital $(r=-.30, p<.05)$, satisfacción laboral $(r=-.36$, $p<.01)$ y engagement $(r=-.44, p<.01)$. El CA mostraba una relación significativa con las siguientes variables: afecto positivo $(r=.28 p<.05)$ $\mathrm{y}$ engagement $(r=.36, p<.01)$. El CN se asociaba de forma significativa a afecto positivo $(r=.34 ., p<.01)$, satisfacción laboral $(r=.26, p<.05)$ y engagement $(r=.32, p<.01)$. El CC relativo a la falta de alternativas se asociaba de forma significativa a todas las variables relativas al bienestar (afecto positivo $(r=-.33, p<.01)$, afecto negativo $(r=.42, p<.01)$, satisfacción vital $(r=-.31, p<.05)$, satisfacción laboral $(r=-.30, p<.05)$, y engagement $(r=-.35, p<.01)$. En cambio, el relativo al sacrificio no se asociaba de forma significativa con ninguna de estas variables.

Para profundizar en mayor medida en las relaciones entre el sentido de pertenencia, los diferentes tipos de compromiso y las diversas variables utilizadas para evaluar el bienestar psicológico se llevaron a cabo análisis de regresión donde el sentido de pertenencia y los diferentes tipos de compromiso eran introducidos como predictores. Los resultados para el grupo de agentes (Tabla 3, parte superior) mostraron que en la predicción del afecto positivo, el sentido de pertenencia $(\beta=-.16, p<.01)$, el CA $(\beta=.31, p<.01)$, el CN $(\beta=.26, p<.01)$ y el CC de falta de alternativas eran predictores significativos $(\beta=-.10, p<.01)$, siendo el CA el más importante de todos. Con relación a la predicción del afecto negativo, solo el sentido de pertenencia $(\beta=.17, p<.01)$ y el CC de falta de alternativas resultan significativos $(\beta=.12, p<.01)$. Para la satisfacción vital, el CA $(\beta=.22, p<.01)$ y el relativo a falta de alternativas $(\beta=-.12, p<.05)$ son los únicos que resultan significativos. En la satisfacción laboral, el sentido de pertenencia $(\beta=-.13, p<.01)$, el CA $(\beta=.60, p<.01)$ y el CCF resultan ser predictores significativos $(\beta=-.10, p<.05)$. Finalmente, el CA fue el único predictor significativo del engagement $(\beta=.65, p<.01)$. 
Los resultados para el grupo de mandos (Tabla 3, parte inferior) mostraron que el sentido de pertenencia era el único predictor significativo del afecto positivo $(\beta=-.41, p<.01)$, del afecto negativo $(\beta=.37$, $p<.01)$ y del engagement $(\beta=-.36, p<.01)$. En el resto de los casos no se encontró ninguna variable que tuviese un peso predictivo significativo.

Cuando se atiende a los porcentajes de varianza explicada se pueden comprobar importantes diferencias en función del puesto de los empleados. En los dos casos, la capacidad predictiva de estos constructos es bastante limitada con relación a la satisfacción vital. Para los empleados agentes teleoperadores, los constructos tienen una mayor capacidad predictiva de engagement y de la satisfacción laboral en comparación con los empleados con puestos de mando. En cambio, en estos últimos se encuentra una mayor capacidad predictiva del afecto negativo.

\section{Discusión}

Los resultados hallados permiten apoyar la validez discriminante del sentido de pertenencia y los diferentes tipos de compromiso organizacional. En la mayoría de los casos los constructos se muestran como relacionados, con la excepción del compromiso de continuidad basado en el sacrificio. Estos resultados son coherentes con lo previamente hallado por Dávila y Jiménez (2012) respecto al CA.

Con relación a la asociación de estos constructos con los indicadores de bienestar para los empleados de base, el sentido de pertenencia se asocia de forma significativa con todos los indicadores, al igual que lo hacen prácticamente en todos los casos los diferentes tipos de compromiso organizacional. En base a las magnitudes de tales asociaciones, el CA sería en líneas generales el constructo que muestra una mayor asociación con el bienestar de los empleados. En cambio, para los empleados con puestos de mando el compromiso organizacional no parece tener la misma importancia. El sentido de pertenencia es el constructo que parece tener la vinculación más fuerte con el bienestar. 


\section{Tabla 3}

Resumen de los análisis de regresión para predecir el bienestar

AGENTES

\begin{tabular}{lcccccccccccccccccc}
\hline & \multicolumn{1}{c}{ Afecto +} & \multicolumn{1}{c}{ Afecto -} & \multicolumn{3}{c}{ Satisfacción V. } & Satisfacción L. & \multicolumn{2}{c}{ Engagement } \\
\hline Variables & B & SE B & $\beta$ & B & SE B & $\beta$ & B & SE B & $\beta$ & B & SE B & $\beta$ & B & SE B & $\beta$ \\
\hline $\begin{array}{l}\text { S. de } \\
\text { pertenencia }\end{array}$ & -.17 & .05 & $-.16^{* *}$ & .17 & .05 & $.17^{* *}$ & -.10 & .07 & -.08 & -.17 & .05 & $-.13^{* *}$ & -.09 & .05 & -.08 \\
CA & .21 & .05 & $.31^{* *}$ & -.10 & .05 & -.10 & .19 & .07 & $.22^{* *}$ & .55 & .05 & $.60^{* *}$ & .50 & .04 & $.65^{* *}$ \\
CN & .20 & .05 & $.26^{* *}$ & .01 & .06 & .01 & .07 & .08 & .07 & .04 & .06 & .04 & .07 & .05 & .08 \\
CCF & -.07 & .03 & $-.10^{*}$ & .12 & .04 & $.12^{* *}$ & -.10 & .05 & $-.12^{*}$ & -.10 & .04 & $-.10^{*}$ & -.04 & .03 & -.05 \\
CCS & -.04 & .04 & -.06 & -.00 & .05 & -.00 & -.10 & .06 & -.12 & .06 & .05 & .06 & -.06 & .04 & -.07 \\
\hline$R^{2}$ & & .32 & & & .11 & & & .08 & & & .54 & & & .49 & \\
\hline MANDOS & & & & & & & & & & & & & & \\
\hline
\end{tabular}

Nota: ${ }^{*} p<.05 ;{ }^{* *} p<.01$

Los resultados derivados de las ecuaciones de regresión van en la misma línea que los que se acaban de describir, pero permiten clarificar la importancia relativa de cada constructo. En este sentido, con relación a los agentes el CA es el factor más relevante de cara a la explicación del bienestar. En cambio, para los mandos solo el sentido de pertenencia predice de forma significativa algunos de los indicadores del bienestar. 
Quizás la causa de las diferencias en estos resultados se base en los diferentes niveles de compromiso experimentado por cada uno de los grupos de empleados. Cuando mayor es el nivel de compromiso, otros constructos como el sentido de pertenencia, puede que pasen a tener un mayor poder de influencia en la predicción del bienestar. En este sentido, el compromiso funcionaría como una actitud hacia la organización previa y necesaria que los trabajadores deben experimentar antes de que cobren importancia otros factores como es el caso del sentido de pertenencia. En línea con esta hipótesis, un elemento importante a tener en cuenta es que en el caso de los mandos generalmente se llega a este tipo de puestos a través de promoción interna y tras un tiempo de permanencia amplio en la categoría inferior, es decir, como teleoperador. Esto supone que previamente se ha desarrollado un compromiso suficiente como para desear permanecer y crecer dentro de la organización, y es tras esta fase cuando quizás sea más probable que se desarrolle una mayor identificación con la organización, y concretamente un mayor sentido de pertenencia.

Con relación a los diferentes tipos de compromiso, los resultados hallados con los trabajadores de base van en la misma línea que lo aportado en estudios previos: en el CA se obtienen resultados coherentes con lo hallado por Zickar y colaboradores (2004) y Lu, Siu, Spector y Shi (2009) en cuanto a la satisfacción vital, y Thorensen y colaboradores (2003) en cuanto al afecto positivo y negativo. El CN solo es un predictor significativo del afecto positivo aunque presenta una correlación positiva y significativa con cada uno de los indicadores del bienestar estudiados. Con relación al CC basado en el sacrificio, este no predice de forma significativa ninguna variable del bienestar, lo que sería coherente con lo hallado por Panaccio y Vandenberghe (2009), aunque se asocia de forma positiva y significativa con el afecto positivo, la satisfacción laboral y el engagement. Finalmente, en el caso del CC basado en la falta de alternativas, este aparece como un predictor significativo del afecto positivo y negativo, de la satisfacción vital y de la satisfacción laboral, y mantiene relaciones significativas con todos los indicadores del bienestar estudiados. Por lo que los resultados en este último caso serían coherentes con lo hallado por otros autores (Meyer et al., 2002; Panaccio \& Vandenberghe, 2008; Zickar et al., 2004). 
Panaccio y Vandenberghe (2008) describen que el CA tendría una mayor vinculación con el bienestar en base a la teoría de la autodeterminación (Deci \& Ryan, 1985), según la cual la regulación interna o intrínseca se asociaría con la experiencia de un mayor bienestar. El CA sería el tipo de compromiso más regulado internamente ya que se basa en el deseo de permanecer en la organización. A su vez, el CN refleja una obligación percibida que puede llevar a una internalización de la presión normativa que ejerce la organización. Cuando el $\mathrm{CN}$ es experimentado como un imperativo moral se puede asociar con una regulación intrínseca y se relacionaría positivamente al bienestar subjetivo y psicológico, pero cuando se experimenta como una deuda con la organización, la regulación deja de ser intrínseca o autónoma, lo que puede hacer que el CN llegue a estar menos intrínsecamente regulado que el CA y, por tanto, su relación con el bienestar será menos intensa en comparación con la establecida con el CA. Por otro lado, el CC es un tipo de compromiso regulado por contingencias externas (percepción de elevados costes por dejar la organización o percepción de tener demasiadas pocas alternativas de empleo) y, por tanto, también lleva a que esté menos regulado intrínsecamente que el CA y que su influencia sobre el bienestar sea menor. A su vez, atendiendo a las diferencias entre bienestar subjetivo y psicológico, Ryan y colaboradores (2008) describen que mientras el bienestar psicológico requeriría la experiencia de una regulación interna o autónoma, el bienestar subjetivo, al menos a corto plazo, podría desarrollarse en base a condiciones de regulación externa, algo que podría explicar la nula capacidad predictiva del $\mathrm{CN}$ y del CC del engagement y que el CA sea su único predictor significativo en el presente estudio en el caso de los trabajadores de base, algo coherente con los resultados aportados por Hallberg \& Schaufeli (2006).

Meyer y Malti (2010) describen también que el efecto del compromiso sobre el bienestar puede tener otras potenciales explicaciones, como por ejemplo, que el compromiso amortigüe el efecto de ciertos estresores, haciendo menos probable que los trabajadores los experimenten o generando recursos como el apoyo social que les ayuden a afrontarlos positivamente. También podría darse lo que se ha dado 
en llamar la paradoja del compromiso, en la que el compromiso en sí mismo tiene potenciales beneficios sobre la salud pero, bajo algunas condiciones, pueda llevar a incrementar la vulnerabilidad de los empleados a los estresores laborales (Galais \& Moser, 2009). A pesar de ello, cuando esto ocurre, el estrés experimentado por los empleados con un fuerte CA no excede del experimentado por aquellos con un compromiso débil.

Con relación al sentido de pertenencia, como un aspecto propio de la identificación organizacional, los resultados hallados serían en gran medida coherentes con los aportados por Wegge y colaboradores (2006), que encontraron que la identificación organizacional era un predictor del bienestar, y también irían en la misma línea que los hallados por otros autores específicamente en torno al sentido de pertenencia, aunque no sean desarrollados en el ámbito laboral (Kitchen et al., 2012, por ejemplo). Por otro lado, en base a los resultados obtenidos resulta difícil identificar si puede jugar un papel más importante en la predicción del bienestar psicológico o del bienestar subjetivo, ya que la importancia de sus vinculaciones depende en gran medida del grupo de trabajadores estudiados, aunque en los dos casos se muestra ineficaz para predecir la satisfacción vital. Como potenciales explicaciones de la influencia del sentido de pertenencia sobre el bienestar, Wegge y su equipo (2006) describieron que una fuerte vinculación con la organización podría ayudar a los trabajadores a adherirse a las normas organizacionales y eso les llevaría a manejar los problemas asociados a sus puestos de forma más eficiente. Finalmente, la experiencia psicológica del sentido de pertenencia podría contribuir al bienestar al permitir satisfacer una necesidad humana considerada básica (Maslow, 1954).

En cuanto a los diferentes resultados hallados para cada uno de los grupos de trabajadores, se debe tener presente las diferentes características de los puestos desarrollados por cada uno de ellos. Tal y como se ha reflejado en Wegge y colaboradores (2006), los puestos de agente teleoperador en call centre son muy demandantes con relación a diversos aspectos, como por ejemplo, la necesidad de realizar correctamente diversas tareas al mismo tiempo (mantener una conversación fluida y 
correctamente, seguir unas pautas de comunicación, introducir datos en el ordenador, etc.), la necesidad de controlar constantemente las propias emociones, lo que puede llegar a generarles disonancia emocional, o la poca autonomía experimentada a la hora de desarrollar su trabajo. Además, implican una alta exigencia de cumplimiento de normas y consecución de resultados, ligados generalmente a su retribución económica y a su permanencia en la organización. En cambio, los puestos de mando pueden llegar a ser también demandantes por la responsabilidad que deben asumir al dirigir, controlar y supervisar el trabajo de los subordinados. En estos puestos se debe hacer cumplir a los subordinados las normas de comportamiento que marca este tipo de trabajo, que suelen implicar una alta disciplina en aspectos de suma importancia para el buen funcionamiento del servicio o departamento en el que se trabaja (tiempos de conexión y desconexión, lo que implica solicitar permiso para realizar cualquier conducta que implique dejar de trabajar, como por ejemplo hacer un pequeño descanso o ir al baño; control del número de tareas realizadas, ya sean llamadas emitidas, llamadas atendidas, documentos revisados, etc.; control del riguroso cumplimiento del guión de conversación o argumentario que tienen que seguir en la conversación con el cliente, etc.). Además, tienen que actuar como interlocutores atendiendo a las demandas de los puestos superiores y los puestos inferiores, lo que puede generar la experiencia de muy diferentes tipos de estrés (ambigüedad de rol, conflicto de rol, etc.). Quizás el análisis exhaustivo de las diferencias entre estos dos tipos de puestos pueda dar la clave de qué determina que en un caso el compromiso sea más relevante y en el otro caso lo sea el sentido de pertenencia.

Respecto a las implicaciones prácticas de los resultados obtenidos, partiendo de la idea de que el bienestar de los empleados genera claras consecuencias positivas para el funcionamiento y resultados de las organizaciones, es obvio que desarrollar políticas de gestión de personas que favorezcan el compromiso y el sentido de pertenencia es una herramienta fundamental para el éxito. Conseguir una cadena de mando con un alto sentido de pertenencia, no solo asegurará una mayor exigencia en el cumplimiento de las normas de la organización, sino que esto irá 
ligado a un mayor bienestar y a una mejora de los resultados generales del equipo de trabajo. Para ello, es recomendable generar prácticas que ayuden a desarrollar la percepción de apoyo de los compañeros, relaciones interpersonales saludables y la cohesión del equipo de mandos. En el caso de las políticas de gestión que se deben desarrollar en los trabajadores de base con el objetivo de generar bienestar, deberían ir más dirigidas a fomentar el compromiso a través, por ejemplo, de promover la congruencia de valores entre el empleado y la organización, y la percepción de apoyo organizacional (Dávila \& Jiménez, 2012).

Como limitaciones del estudio, se observa el reducido tamaño de la muestra de empleados que desempeñan puestos considerados de mando, lo que ha podido llevar a que el poder de los análisis estadísticos sea limitado, y esto puede haber contribuido a subestimar la influencia de algunas variables, como por ejemplo, los diferentes tipos de compromiso organizacional. Sumado a ello, el estudio se ha basado por completo en medidas de auto-reporte. El interés no se centraba tanto en obtener una medida fiable de los conceptos estudiados, sino en recoger la percepción de las personas en cada aspecto. Otra limitación ha sido el uso de un diseño transversal por sus implicaciones a la hora de establecer relaciones de causalidad. Por último, el hecho de que la muestra proceda de una única organización que realiza fundamentalmente un tipo de servicio, puede haber tenido algún efecto sobre la varianza observada en la medición de algunas variables.

A la luz de los resultados obtenidos no se puede descartar un constructo en beneficio de otro. El compromiso organizacional y el sentido de pertenencia aluden a dos constructos diferentes, y tanto uno como otro pueden ser especialmente relevantes en la predicción de ciertas consecuencias en función de algunas variables contextuales, como por ejemplo el tipo de puesto. En base a este trabajo consideramos que sería deseable que el sentido de pertenencia se considerase como una faceta adicional para el análisis de la vinculación psicológica con la organización y, en este sentido, también sería deseable profundizar en las relaciones de causalidad que pueden establecerse entre las diferentes facetas, por ejemplo Galais y Moser (2009) describen que el 
compromiso organizacional cultiva o nutre la necesidad de pertenencia, y en el papel mediador que pueden jugar cada una de ellas en la predicción de otras variables por parte de cada una.

\section{Referencias}

Addae, H. M. \& Wang, X. (2006). Stress at work: Linear and curvilinear effects of psychological -,job,- and organizational-related factors: An exploratory study of Trinidad y Tobago. International Journal of Stress Management, 13, 476-493. http://dx.doi. org/10.1037/1072-5245.13.4.476

Anant, S. S. (1966). The need of belong. Canada's Mental Health, 14, 21-27.

Ashforth, B. E. \& Mael, F. (1989). Social identity theory and the organization. Academy of Management Journal, 14, 20-39. http:// dx.doi.org/10.5465/amr.1989.4278999

Atienza, F. L., Pons, D., Balaguer, I. \& Garcia-Merita, M. L. (2000). Psychometric properties of Satisfaction with Life Scale in adolescents. Psicothema, 12, 331-336.

Bakker, A., Demerouti, E., de Boer, E. \& Schaufeli, W. B. (2003). Job demands and job resources as predictors of absence duration and frequency. Journal of Vocational Behavior, 62, 341-356. http:// dx.doi.org/10.1016/S0001-8791(02)00030-1

Bentein, K., Vandenberg, R. J., Vandenberghe, C. \& Stinglhamber, F. (2005). The role of change in the relationship between commitment and turnover: A latent growth modeling approach. Journal of Applied Psychology, 90, 468-482. http://dx.doi.org/10.1037/ 0021-9010.90.3.468

Blanco, A. \& Díaz, D. (2005). El bienestar social: su concepto y medición. Psicothema, 17(4), 582-589.

Bridger, R. S., Kilminster, S. \& Slaven, G. (2007). Occupational stress and strain in the naval service: 1999 and 2004. Occupational Medicine, 57, 92-97. http://dx.doi.org/10.1093/occmed/kql124 
Brotheridge, C. M. \& Lee, R. T. (2003). Development and validation of the emotional labour scale. Journal of Occupational and Organizational Psychology, 76, 365-379. http://dx.doi.org/ 10.1348/096317903769647229

Cambré, B., Kippers, E., van Veldhoven, M. \& de Witte, H. (2012). Jobs and organisations: Explaining group level differences in job satisfaction in the banking sector. Personnel Review, 41(2), 200-215. http://dx.doi.org/10.1108/00483481211200033

Cameron, J. E. (2004). A three-factor model of social identity. Self and Identity, 3, 239-262. http://dx.doi.org/10.1080/ 13576500444000047

Cooper-Hakim, A. \& Viswesvaran, C. (2005). The construct of work commitment: testing and integrative Framework. Psychological Bulletin, 131, 241-259. http://dx.doi.org/10.1037/ 0033-2909.131.2.241

Culberton, S. S., Fullagar, C. J. \& Mills, M. J. (2010). Feeling good and doing great: the relationship between psychological capital and well-being. Journal of occupational health psychology, 15(4), 421-433. http://dx.doi.org/10.1037/a0020720

Danna, K. \& Griffin, R. W. (1999). Health and well-being in the workplace. A review and synthesis of the literature. Journal of Management, 25(3), 357-384. http://dx.doi.org/10.1177/ 014920639902500305

Dávila, M. C. \& Jiménez, G. (2012). Organizational identification and commitment: correlatos of sense of belonging and affective commitment. The Spanish Journal of Psychology, 15(1), 244-255. http://dx.doi.org/10.5209/rev_SJOP.2012.v15.n1.37316

Deci, E. L. \& Ryan, R. M. (1985). Intrinsic motivation and self-determination in human behavior. New York: Plenum. http://dx.doi. org/10.1007/978-1-4899-2271-7

Díaz, D., Rodríguez-Carvajal, R., Blanco, A., Moreno-Jiménez, B., Gallardo, I., Valle, C. \& van Dierendonck, D. (2006). Adaptación española de las escalas de bienestar psicológico de Ryff. Psicothema, 18(3), 572-577. 
Díaz, J. F. \& Sánchez-López, M. P. (2002). Relaciones entre estilos de personalidad y satisfacción autopercibida en diferentes áreas vitales. Psichotema, 14(1), 100-105.

Diener, E., Emmons, R., Larsen, R. \& Griffin, S. (1985). The satisfaction with Life Scale. Journal of Personality Assessment, 49(1), 71-75. http://dx.doi.org/10.1207/s15327752jpa4901_13

Diener, E., Suh, E. M., Lucas, R. E. \& Smith, H. L. (1999). Subjective well-being: Three decades of progress. Psychological Bulletin, 125, 2, 276-302. http://dx.doi.org/10.1037/0033-2909.125.2.276

Epitropaki, O. \& Martin, R. (2005). From ideal to real: A longitudinal study of the role of implicit leadership theories on leader-member exchanges and employee outcomes. Journal of Applied Psychology, 90, 659-676. http://dx.doi.org/10.1037/0021-9010.90.4.659

Galais, N. \& Moser, K. (2009). Well-being of temporary agency workers: A longitudinal study. Human Relations, 62(4), 589-620. http://dx.doi.org/10.1177/0018726708101991

Gautam, T., Van Dick, R. \& Wagner, U. (2004). Organizational identification and organizational commitment: Distinct aspects of two related Concepts. Asian Journal of Social Psychology, 7, 301-315. http://dx.doi.org/10.1111/j.1467-839X.2004.00150.x

Grawitch, M. J., Trares, S. \& Kohler, J. M. (2007). Healthy workplace practices and employee outcomes. International Journal of Stress Management, 14, 275-293. http://dx.doi.org/10.1037/10725245.14.3.275

Hagerty, B. M., Wiliams, R. A., Conye, J. C. \& Early, M. R. (1996). Sense of belonging and indicators of social and psychological functioning. Archives of Psychiatric Nursing, 10(4), 235-244. http://dx.doi.org/10.1016/S0883-9417(96)80029-X

Hagerty, B. M. K. \& Patusky, K. (1995). Developing a measure of sense of belonging. Nursing Research, 44(1), 9-13. http://dx.doi. org/10.1097/00006199-199501000-00003

Hagerty, B. M. K., Lynch-Sauer, J., Patusky, K. L., Bouwsema, M. \& Collier, P. (1992). Sense of belonging: A vital health concept. Archives of Psychiatric Nursing, 6(3), 172-177. http://dx.doi.org/ 10.1016/0883-9417(92)90028-H 
Hallberg, U. E. \& Schaufeli, W. B. (2006). "Same same: but different: Can work engagement be discriminated from job involvement and organizational commitment? European Journal of Psychology, 11, 119-127. http://dx.doi.org/10.1027/1016-9040.11.2.119

Harris, G. E. \& Cameron, J. E. (2005). Multiple dimensions of organizational identification and commitment as predictors of turnover intentions and psychological well-being. Canadian Journal of Behavioural Science, 37(3), 159-169. http://dx.doi. org/ $10.1037 / \mathrm{h} 0087253$

Irving, P. G. \& Coleman, D. F. (2003). The moderating effect of different forms of commitment on role ambiguity -job tension relations. Canadian Journal of Administrative Sciences, 20, 97-106. http:// dx.doi.org/10.1111/j.1936-4490.2003.tb00696.x

Johnson, D. R., Alvarez, P., Longerbeam, S., Soldner, M., Kurotsuchi, K., Brown, J. \& Rowan-Kenyon, H. (2007). Examining sense of belonging among first-year undergraduates form different racial/ethnic groups. Journal of College Student Development, 48(5), 525-542. http://dx.doi.org/10.1353/csd.2007.0054

Kavanagh, M. J. \& Halpern, M. (1977). The impact of job level and sex differences on the relationship between life and job satisfaction. Academy of Management Journal, 20(1), 66-73. http:// dx.doi.org/10.2307/255462

Kitchen, P., Williams, A. \& Chowhan, J. (2012). Sense of community belonging and health in Canada: A regional analysis. Social Indicators Research, 107(1), 103-126. http://dx.doi.org/10.1007/ s11205-011-9830-9

Lambert, E. G., Hogan, N. L. \& Griffin, M. L. (2008). Being the good soldier: Organizational citizenship behavior and commitment among correctional staff. Criminal Justice and Behavior, 35, 56-68. http://dx.doi.org/10.1177/0093854807308853

Lisbona, A., Morales, J. F. \& Palací, F. J. (2006). Identidad y compromiso en equipos de intervención en emergencias. Psicothema, 18(3), 407-412. 
Lu, J. F., Siu, O. L., Spector, P. E. \& Shi, K. (2009). Antecedents and outcomes of a fourfold taxonomy of work-family balance in Chinese employed parents. Journal of Occupational Health Psychology, 14, 182-192. http://dx.doi.org/10.1037/a0014115

Maslow, A. (1954). Motivation and personality. New York: Harper.

McCallum, C. \& McLaren, S. (2011). Sense of belonging and depressive symptoms among GLB adolescents. Journal of Homosexuality, 58(1),83-96.http://dx.doi.org/10.1080/00918369.2011.533629

Meyer, J. P., Allen, N. J. \& Topolnysky, L. (1998). Commitment in a changing world of work. Industrial-Organizational Psychology and Emerging Needs of the Canadian Workplace, 39(1-2), 83-93.

Meyer, J. P. \& Allen, N. J. (1991). A three-component conceptualization of organizational commitment. Human Resource Management Review, 1, 61-89. http://dx.doi.org/10.1016/ 1053-4822(91)90011-Z

Meyer, J. P. \& Maltin, E. R. (2010). Employee commitment and wellbeing: A critical review, theoretical framework and research agenda. Journal of Vocational Behaviour, 77, 323-337. http:// dx.doi.org/10.1016/j.jvb.2010.04.007

Meyer, J. P., Stanley, D. J., Herscovitch, L. \& Topolnytsky, L. (2002). Affective, continuance, and normative commitment to the organization: A meta-analysis of antecedents, correlates, and consequences. Journal of Vocational Behavior, 61(1), 20-52. http://dx.doi.org/10.1006/jvbe.2001.1842

Mock, S. E., Fraser, C., Knutson, S. \& Prier, A. (2010). Physical leisure participation and the well-being of adults with rheumatoid arthritis: The role of sense of belonging. Activities, Adaptation \& Aging, 34(4), 292-302. http://dx.doi.org/10.1080/01924788.2 010.523870

Mullarkey, S., Jackson, P. R., Wall, T. D., Wilson, J. R. \& Grey-Taylor, S. M. (1997). The impact of technology characteristics and job control on worker mental health. Journal of Organizational Behaviour, 18, 471-489. http://dx.doi.org/10.1002/(SICI)10991379(199709)18:5<471::AID-JOB810>3.0.CO;2-V 
Panaccio, A. \& Vandenberghe, C. (2008). Perceived organizational support, organizational commitment and psychological wellbeing: A longitudinal study. Journal of Vocational Behaviour, 75, 224-236. http://dx.doi.org/10.1016/j.jvb.2009.06.002

Riketta, M. (2005). Organizational identification: A meta-analysis. Journal of Vocational Behavior, 66, 358-384. http://dx.doi.org/ 10.1016/j.jvb.2004.05.005

Ron, Y. (2004). No man is an island: The relationship between attachment styles, sense of belonging, depression and anxiety among homeless adults. ProQuest Information \& Learning, AAI3133630.

Ryan, R. M., Huta, V. \& Deci, E. L. (2008). Living well: A self-determination theory perspective on eudaimonia. Journal of Happiness Studies, 9, 139-170. http://dx.doi.org/10.1007/s10902-0069023-4

Ryan, R. \& Deci, E. (2001). On happiness and human potentials: a review of research on hedonic and eudaimonic well-being. Annual Review of Psychology, 52, 141-166. http://dx.doi.org/ 10.1146/annurev.psych.52.1.141

Salanova, M. \& Schaufeli, W. (2009). El engagement en el trabajo: Cuando el trabajo se convierte en pasión. Madrid: Editorial Alianza.

Schaubroeck, J. \& Jones, J. R. (2000). Antecedents of workplace emotional labor dimensions and moderators of their effects on physical symptoms. Journal of Organizational Behavior, 21, 163-183. http://dx.doi.org/10.1002/(SICI)10991379(200003)21:2<163::AID-JOB37>3.0.CO;2-L

Schaufeli, W. B., Bakker, A. \& Salanova, M. (2006). The Measurement of Work Engagement With a Short Questionnaire: A CrossNational Study. EducationalandPsychologicalMeasurement, 66(4), 701-716. http://dx.doi.org/10.1177/0013164405282471

Siu, O. (2002). Occupational stressors and well-being among Chinese employees: The role of organizational commitment. Applied Psychology: An International Review, 51, 527-544. http://dx.doi. org/10.1111/1464-0597.t01-1-00106 
Somers, M. J. (2009). The combined influence of affective, continuance, and normative commitment on employee withdrawal. Journal of Vocational Behavior, 74, 75-81. http://dx.doi.org/10.1016/ j.jvb.2008.10.006

Stinglhamber, F., Bentein, K. \& Vandenberghe, C. (2002). Extension of the three-component model of commitment to five foci: Development of measures and a substantive test. European Journal of Psychological Assessment, 18, 123-138. http://dx.doi. org/10.1027//1015-5759.18.2.123

Tajfel, H. \& Turner, J. C. (1979). An integrative theory of intergroup conflict. InW. G. Austin \& S. Worchel (Eds.), The social psychology of intergroup relations (pp. 33-47). Monterey: Brooks/Cole.

Tan, D. S. K. \& Akhtar, S. (1998). Organizational commitment and experienced burnout: An exploratory study from a Chinese cultural perspective. International Journal of Organizational Analysis, 6, 310-333. http://dx.doi.org/10.1108/eb028889

Thorensen, C. J., Kaplan, S. A., Barsky, A. P., Warren, C. R. \& de Chermont, K. (2003). The affective underpinning of job perceptions and attitudes: A meta-analytic review and integration. Psychological Bulletin, 129, 914-945. http://dx.doi. org/10.1037/0033-2909.129.6.914

Van Dick, R. \& Wagner, U. (2002). Social identification among school teachers: Dimensions, foci, and correlates. European Journal of Work and Organizational Psychology, 11, 129-149. http://dx.doi. org/10.1080/13594320143000889

Van Knippenberg, D. \& Sleebos, E. (2006). Organizational identification versus organizational commitment: Self-definition, social Exchange, and job attitudes. Journal of Organizational Behavior, 27, 571-584. http://dx.doi.org/10.1002/job.359

Warr, P., Cook, J. \& Wall, T. (1979). Scales for the measurement of some work attitudes and aspects of psychological well-being. Journal of Occupational Psychology, 52, 129-148. http://dx.doi. org/10.1111/j.2044-8325.1979.tb00448.x 
Watson, D., Clark, L. A. \& Tellegen, A. (1988). Development and validation of brief measures of positive and negative affect: The PANAS scales. Journal of Personality and Social Psychology, 54, 1063-1070. http://dx.doi.org/10.1037/0022-3514.54.6.1063

Wegge, J., van Dick, R., Fisher, G. K., Wecking, C. \& Moltzen, K. (2006). Work motivation, organizational identification, and well-being in call centre work. Work \& Stress, 20(1), 60-83. http://dx.doi.org/10.1080/02678370600655553

Young, A. F., Russell, A. \& Powers, J. R. (2004). The sense of belonging to neighbourhood: can it be measured and is it related to health and well being in older women? Social Science \& Medicine, 59, 2627-2637. http://dx.doi.org/10.1016/j.socscimed.2004.05.001

Zickar, M. J., Gibby, R. E. \& Jenny, T. (2004). Job attitudes of workers with two jobs. Journal of Vocational Behavior, 64, 222-235. http://dx.doi.org/10.1016/S0001-8791(03)00047-2.

Recibido: 27 de mayo, 2014 Aceptado: 30 de junio, 2014 